the Solar System, in which case this planet also represents the extreme case of planetary modification by collision - a collision that only just fell short of complete fragmentation.

The purpose of the book reviewed here is to revive interest in Mercury, more than a decade after the Mariner 10 fly-bys. Like other volumes in the highly successful University of Arizona Press Space Science series, it stems from a conference, in this case one held in August 1986. But this is not simply a book of conference proceedings. It is a comprehensive, carefully edited volume, and as well as the 23 chapters (by a total of 47 contributors) it includes an extensive, integrated bibliography, a glossary and an index.

Clark Chapman and Faith Vilas, two of the editors, have written the first two chapters, presenting an excellent overview of the planet and discussing future observations and missions. Until recently Mercury had not been considered as a target by the National Aeronautics and Space Administration, the European Space Agency and the Soviet Union, but things may be changing, as clever new ways have been found to use multiple gravity assists from Venus and Mercury itself to manoeuvre an orbiter or lander craft to Mercury. Scientifically exciting missions are therefore possible with existing launch vehicles (Titan/Centaur, Ariane V, or Proton); but it remains to be seen how the priorities for missions to Mercury will rank in comparison to those for Mars, Saturn or comets in a period of limited spending for space science and exploration.

Meanwhile this book provides an excellent summary of our current knowledge of the planet. Several contributors discuss its geology, comparing its history of impact cratering and volcanism with that of the Moon, which it superficially resembles. Mercury's unique compressional scarps tell a tale of internal shrinking due to cooling and phase changes, while the presence of a global magnetic field requires a partially liquid core and places constraints on bulk composition. Also discussed are the recently discovered atmosphere of sodium and potassium, and interactions between the magnetic field of the planet and the solar wind. Perhaps most interesting are the chapters that synthesize the data on composition and structure to develop theories of Mercury's formation and evolution, including the giant impact hypothesis for removal of much of its silicate shell. The treatment is authoritative and comprehensive, providing within one cover very nearly all we know - although certainly less than planetary scientists would like to know - about this remarkable planet.

David Morrison is Chief of the Space Science Division, NASA Ames Research Center, Moffett Field, California 94035, USA.

\section{Analysis under analysis}

Michael B. Richman

Principal Component Analysis in Meteorology and Oceanography. By Rudolph W. Preisendorfer. Posthumously compiled and edited by Curtis D. Mobley. Elsevier: 1988. Pp.425. Dfl.210.

Principal component analysis (PCA) is a powerful multivariate technique that can be applied to a range of problems in the social and physical sciences. It has roots in fields as diverse as statistics, physics, biology, psychology and meteorology, and has undergone parallel evolution in each of them.

Rudolph Preisendorfer's ability to assimilate and distil information from a wide array of subject areas and identify a common denominator is revealed in this book. It is a predominantly theoretical text, with enough applied material to help the reader grasp the importance of the derivational work. The first four chapters outline in detail the algebraic foundations of PCA, and its dynamical origins and multivariate formulations. One difficulty in compiling this type of book, dealing with interdisciplinary aspects of eigenvector research, is the varied and contradictory jargon used - one researcher's factors may be another's components and a third's empirical orthogonal functions (EOFs). Thankfully, at the outset Preisendorfer sets a convention for terminology, deciphering many articles so that their message is not lost.

As well as the derivation of PCA, Section 2 presents the origins of singular value decomposition and demonstrates its importance in bridging PCA and EOFs. Interspersed with the theory are several useful examples to help cement the concepts. Chapter 3 provides classic examples from Preisendorfer's earlier work on the dynamical origins of simple systems to illustrate properties of PCA, which are than generalized to vector fields in Chapter 4.

One of the highlights of the book, found in Chapter 5, is a critical examination of a range of $\mathrm{PC}$ truncation selection rules developed to separate signal from noise. There are several subsections which contain helpful comments, comparisons, and caveats for groups of rules. This chapter, combined with the bibliographical notes, will be required reading for anyone who is interested in gaining a full understanding of both the older and current methods of separating signal from noise in eigenanalyses.

The next few chapters examine connections between PCA and the related techniques of factor analysis (FA), canonical correlation analysis (CCA) and linear regression analysis (LRA). Much of the material on FA is fashioned after Lawley and Maxwell's Factor Analysis as a Statistical Method (Butterworths; 2nd Edn 1971), and shares their embracement of the maximum likelihood method. The relevant bibliographical notes are well worth turning to for added insight, though readers wanting a full view of factor methods and problems associated with parameter estimation and indeterminacy will need also to refer to texts such as Mulaik's The Foundations of Factor Analysis (McGraw Hill, 1972).

Chapter 7 provides an introduction to linear transformations which can be applied to either PCA or FA. Preisendorfer cites work by Buell as the reason for going beyond unrotated PCA solutions with specific algorithms (Varimax and Procrustes rotations) being discussed in detail. Again, because this chapter is intended as an introduction to the topic of linear transformations, a few nuances are missing; for example there is no discussion of the role of hyperplanes (as contrasted to clusters) or that of pairwise graphical plots in explaining and validating simple structure. Yet the chapter does offer several glimpses of techniques that are only now beginning to be used by geophysical scientists, for example Procrustes Target Analysis and extended applications of Rule $\mathrm{N}$ with the Principle of Mimicry. The derivation of CCA in a PCA framework (Chapter 8) facilitates understanding of an aspect of CCA in applied research, and there is also a section on CCA selection rules which contains previously unpublished material. Chapter 9 similarly contains unique discussions of PCA regression, hindcast skill, and how this relates to CCA.

The last three chapters deal with statistical-dynamical models, partitioning eigenvectors and complex harmonic PCA. Chapter 10 is an impressive examination of two examples of substituting eigenmodes into models of atmospheric flow. Likewise, Chapter 12 contains a wealth of information on complex PCA, motivated by the thought that "we may reasonably expect that on combining the techniques of PCA and harmonic analysis, something of greater applicability than each will result for certain diagnostic or predictive tasks".

This is a well-organized and well-written text which draws information from half-adozen fields and brings it together into a concise statement on the theory and geophysical uses of principal components. We should be indebted to Curtis Mobley for bringing it to fruition after Preisendorfer's untimely death.

Michael B. Richman is in the Climate and Meteorology Section of the Illinois State Water Survey, 2204 Griffith Drive, Champaign, IIIinois 61820, USA. 